\title{
THE INFLUENCE OF MULTI-LEVEL COVERAGE ON EFFICIENCY OF AMBULANCES RELOCATION OPTIMIZATION PROCESS BASED ON THE AVL DATA
}

\author{
Čedomir Vasić1, \\ Dejan Rančić', \\ Leonid Stojmenov', \\ Jelena Gavrilović2 \\ Nebojša Arsić3 \\ ${ }^{1}$ University of Niš, \\ Faculty of Electronic Engineering, \\ Niš, Serbia \\ ${ }^{2}$ Singidunum University, \\ Belgrade, Serbia \\ ${ }^{3}$ University of Priština, \\ Faculty of Technical Sciences, \\ Kosovska Mitrovica, Serbia
}

\begin{abstract}
:
The implementation of emergency vehicles relocation, as part of the overall optimization process, looking for some in advance adopted guidelines. One of the most important issues is to decide between single, double or other multiple coverage of service points. A comprehensive algorithm based on the data extracted from huge archive of vehicle moving history is used to analyze the influence of different types of coverage to the efficiency of obtained solutions. In order to perform comparison and evaluation of the quality of semi-optimal solutions, a parameter named savings ratio is used.
\end{abstract}

Key words:

relocation of ambulances, multiple coverage, double coverage, location optimization.

\section{PROBLEM DEFINITION}

A comprehensive project is implemented within the Computer Science department at the Faculty of Electronic Engineering, University of Niš, based on the use of the data generated by AVL (Automatic Vehicle Location) component of the GPS/GPRS (Global Positioning System/ General Packet Radio Service) tracking devices. For the purpose of resource allocation in an emergency medical service, a system for tracking and ambulance fleet relocation was brought into operation. During the practical realization of the project, it was necessary to consider several important aspects related to this well-known problem in the field of the locational optimization. Deterministic approach in process of the redeployment of the static ambulance fleet, assume that we already made decision how many vehicles should ensure coverage to the single demand for service. The multiple coverage means that we make a compromise and in clean and simple single-coverage solution, introduce certain lack of efficiency. The aim of this paper is to quantify the extent to which multiple coverage degrades efficiency of initial solution based on the single demand coverage. To that end, the appropriate parameter named "saving ratio", as a unique coefficient of efficiency of the whole process,

\section{is proposed.}

Correspondence:

Čedomir Vasić

e-mail:

dona@dona.rs 


\section{MATHEMATICAL REPRESENTATION}

As regards the available input data, in particular the main goal of the optimization process, we have several different definitions of this problem. The associated mathematical formulation also differs from case to case. One approach is in the case if we want to find how many of the vehicles are necessary to handle the assigned number of service demands, when the basic limitation is not to extent the presumed maximum time limit to reach the farthest user. MCLP (Maximal Coverage Location Problem) is the model proposed by R. L. Church and C. ReVelle [1] to resolve that kind of problems. Different problem setting is if we want to deploy $\boldsymbol{p}$ available vehicles and the main goal is the minimal total distance to serve all demand points. In this case, we deal with the feet of fixed size.

The multiple coverage can be introduced in mathematical formulation in two main ways: static - introducing simple percentage of areal coverage or dynamically - through complex time dependence related to the probability that the observed vehicle will be used in curtain time frame. Frequently cited static model named "BACOP2" is mathematically defined by K. Hogan and C. Revell [2]. In this model, double coverage of destinations is introduced for the first time and the impact of coverage by second vehicle is balanced using the variable named weighting factor. It is up to the operator in "call-centre" to make a choice between different sizes of this parameter in accordance with the predefined business policy.

Dealing with the probability that the vehicle will be busy during the observed time frame, leads us to the probabilistic approach. Unavoidable is the work of M. Gendreau, G. Laporte and F. Semeta [3] in which DSM (Double Standard Model) is defined to maximize the goal that demand is covered by at least two vehicles, implicitly taking into account the fact that vehicles might become unavailable. The model in particular tries to ensure that certain percentage of the population is covered within $r_{1}$ time units and all demand points need to be covered by at least one vehicle within $r_{2}$ time units, where $r_{1}<r_{2}$.

In our research, we were limited to the deterministic approach, and we focused our efforts on resolving the common problem of location optimization, named as " $p$-median" problem. In a few words, it is a problem to identify $\boldsymbol{p}$-facilities, to minimize distances, required to provide a service to $\boldsymbol{n}$-destinations. According to the fact that this problem is classified as non-deterministic polynomial-time (NP) difficulty, time and memory demands to reach the final solution are not known in advance. Contemporary meta-heuristic methods are used as the common approach to find the semi-optimal solution. This problem is well defined and probably represents one of the most often considered issues in the field of location optimization. Different approaches in solving this problem range from S. L. Hakimi [4], C. ReVelle and R. Swain [5], M. B. Teitz and P. Bart [6], P. J. Densham and G. Rushton [7], E. L. F. Senne and L. A. N. Lorena [8], P. Hansen and N. Mladenović [9], K. E. Rosing and C. S. ReVelle [10], to the contemporary meta-heuristic methods such as Genetic algorithm, described in the works of C. M. Hosage and M. F. Goodchild [11] and O. Alp, E. Erkut and Z. Drezner [12].

The generally adopted representation of $\boldsymbol{p}$-median problem is given as follows: find minimum of (1):

$$
\min \left(\sum_{i \in I} \sum_{j \in J} \omega_{j} \cdot d_{i j} \cdot y_{i j}\right)
$$

where $\omega_{j}$ is a weighting factor of location $\boldsymbol{j}$, while $\boldsymbol{d}_{i j}$ is distance between location $\boldsymbol{i}$ of vehicle dwelling location and location $\boldsymbol{j}$ as one of the observed $\boldsymbol{n}$ service demand locations. In expression (1), $\boldsymbol{I}$ and $\boldsymbol{J}$ are upper limits in the range of the integer variables $\boldsymbol{i}$ and $\boldsymbol{j}$, and it is obvious that in both cases it is the number $\boldsymbol{n}$, according to fact that each of $\boldsymbol{n}$ locations at the same time is a potential candidate for the optimal solution. To gain full and correct mathematical representation of the observed problem, additional constraints are needed and these constraints become an integral part of the definition. In the case of single coverage, additional requirements are:

$$
\begin{gathered}
\sum_{i \in I} x_{i}{ }^{1}=p \\
\sum_{i \in I} y_{i j}=1 \quad \forall j \in J \\
y_{i j}-x_{i}{ }^{1} \leq 0 \quad \forall i \in I, j \in J \\
x_{i}{ }^{1} \in\{0,1\} \quad \forall i \in I \\
y_{i j} \in\{0,1\} \quad \forall i \in I, j \in J
\end{gathered}
$$

Condition (2) is connected with the physical limitations in the number of vehicles and shows that the total number of vehicles needed to be deploed is limited to $p$, but it also requires that we had to deploy all $p$ vehicles. 
Condition (3) defines the assumption that one destination is serviced by only one vehicle and only one parking place is joined to the one demand. If the parking place selected to cover request from location $\boldsymbol{j}$, does not have a vehicle, we have no possible solution and it is described in expression (4). At the same time, that is a way to introduce a single coverage in the definition. The condition (5) defines simple fact that on the single parking place we can park only whole vehicle. It is physically impossible to split one car into two or more locations. A similar fact is described in the condition (6). The intervention is maintained with one whole vehicle, not with two halves of vehicles from two different locations. Conditions (5) and (6) procedure lead into the domain of integer arithmetic, whereby we only had to be careful and ensure that the interpretation of distance $\omega_{j}$ also remains as integer value.

The double coverage we formulate as follows: find a minimum of (7):

$\min \left(\theta \sum_{i \in I} \sum_{j \in J} \omega_{j} \cdot d_{i j} \cdot y_{i j}+(1-\theta) \cdot \sum_{i \in I-1} \sum_{j \in J-1} \omega_{j} \cdot d_{i j} \cdot z_{i j}\right)$ (7)

Expression (7) is obtained from the initial expression (1) in which an extra double sum is added, but restricted to only $\boldsymbol{p}$ - 1 available vehicles, to be allocated to $\boldsymbol{n}$ - 1 locations. Now we have a new requirement, as in one location we can park two vehicles. The conditions (2), (3), (5) and (6) remain, but we had to change the condition (4) and to add new conditions (10) and (11), to define hierarchy between single and double coverage. Relations between single and double coverage are in fact relations between the first and second double sum in expression (7).

$$
\begin{gathered}
\sum_{i \in I-1} x_{i}{ }^{2}=p-1 \\
\sum_{i \in I-1} z_{i j}=1 \quad \forall j \in J \\
z_{i j}-y_{i j} \leq 0 \quad \forall i \in I-1, j \in J-1 \\
z_{i j}-x_{i}{ }^{2} \leq 0 \quad \forall i \in I-1, j \in J-1 \\
x_{i}{ }^{2} \in\{0,1\} \quad \forall i \in I-1 \\
z_{i j} \in\{0,1\} \quad \forall i \in I-1, j \in J-1 \\
0 \leq \theta \leq 1
\end{gathered}
$$

The condition (10) tells that single destination cannot be covered twice, if it is not covered once already, while the condition (11) is extended condition (4). It specifies that the coverage with the second vehicle follows the same logic about possible or impossible solution, already described with expression (4). The objective defined with the equation (7) is a result of the balance between aspiration to cover the observed destination once and aspiration to be covered twice.

The weight factor $\theta$ is introduced to define balance between these two opposite aspirations. Expression (14) says: if value of $\theta$ is closer to 1 , advantage and greater weight has single coverage. If $\theta$ is closer to 0 , it is a growing influence of the double coverage. In the limiting cases, when $\theta=1$, expression (7) is reduced to the formulation (1), and if $\theta=0$, we have also simplification of the problem and definition is switched to complete coverage with two vehicles.

The triple coverage is only further extension of the expressions (1) and (7) and we add an extra double sum, following the same logic that we used to come from single to double coverage. The following expression is labeled as (15):

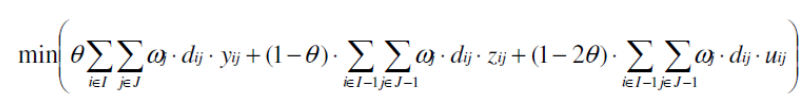

\section{DESCRIPTION OF THE APPLIED METHOD}

Emergency medical service and problems of the allocation and relocation of ambulances is often considered. Some of the most cited and comprehensive efforts are works of P. Kolesar, W. Walker, J. Hausner [13], S. Budge, A. Ingolfsson, D. Zerom [14] and M. Reinthaler, B. Nowotny, F. Weichenmeier [15]. The well documented algorithm of resolving the appropriate $\boldsymbol{p}$-median problem, related to relocation of ambulance vehicles, is described in detail in the previous works of authors of this paper [16], [17], [18]. We proposed an algorithm which is implemented in four independent stages:

I) The first phase is the process of the vehicle destinations extraction from the database which is an integral part of the vehicle tracking system $(\mathrm{AVL})$. Extraction is maintained through execution of several SQL-queries, defined for limited time frame in which fleet of vehicles is observed. In the applied sequential analysis, we use several pre-defined criteria and, we come to the set of $\boldsymbol{n}$ locations which represents destinations of individual drives of ambulances. All these destinations are locations of demands for service in the defined time frame. At the end of the first phase, we construct $n \boldsymbol{n} n$ matrix of distances $M(n, n)$ and 
introduce a weight factor for each destination. In $\boldsymbol{i}$-row and $\boldsymbol{j}$-column a matrix element $\boldsymbol{d}_{\boldsymbol{i}}$ is placed, and that element represents the distance from the destination node $\boldsymbol{i}$ to the destination node $\boldsymbol{j}$ multiplied by $\omega_{j}$-weight factor of node $\boldsymbol{j}$.

II) The second phase of the algorithm is some kind of pre-processing of each element of the matrix of distances $M(n, n)$. Pre-processing assumes that Euclidean distances are replaced with the routed distances. For the purposes of this paper, routing is done using the commercial software "Garmin BaseCamp, Ver.4.3.4, 2008-2014 Garmin Ltd.” [19], which allows tabular input of coordinates and delivers the route. Routes are based on the road network defined by maps labeled as: "City Navigator Europe NT 2014.3 Ver.17.30 NAVTEQ”. The history of vehicle usage analyzed during the first phase of the algorithm contains all information about the routes starting from the Central garage to each destination. However, in the archive there is no information about routing paths between individual destinations. That is the main reason to populate the distance matrix with distances delivered by commercial navigation software.

III) In the third stage of the algorithm, we introduce $\boldsymbol{p}$ service locations as parking places in which we want to set $\boldsymbol{p}$ vehicles. The main objective of this phase is to reach a solution with acceptable quality in an efficient manner. That solution had to be good enough as a starting point for the central pillar of the whole solution and it is the heuristic based on the Genetic algorithm. During the third phase, solution is obtained through direct calculations based on the limited number of iterations where "node-substitution-procedure" is used. During each iteration well known "1-opt-procedure" is performed on the matrix of distances, followed by procedure of finding the nearest neighbors ("neborhooding"). After neighborhooding is done, all destinations become sorted in $\boldsymbol{p}$ subsets, "1-opt-procedure" is used again, but now on every of $\boldsymbol{p}$-subsets. These steps are repeated in several iterations and in the process of direct calculations, we came to the rough solution to each of the $\boldsymbol{p}$ medians. The test example described in this study assumes that the III phase of the algorithm was limited to 20 iterations, after which we get a solution which will be input for the IV phase.

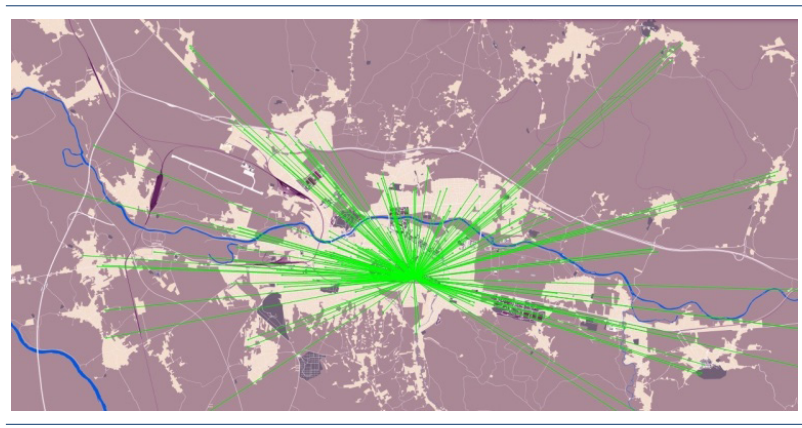

Figure 1. Starting sum of the distances to the 218-destinations $C_{t o t}$.

IV) During phase IV, suitable Genetic algorithm is used and semi-optimal solution is delivered. The specific parameters of proposed Genetic algorithm ensure the rapid convergence and reduce the risks of falling in trap known as local minimum. The execution of the Genetic algorithm is limited to pre-defined number of generations, and for the purpose of this study, we adopted a total number of generations to be restricted to 100. To estimate the quality of the offered relocational solution, we defined a unique parameter to quantify the quality of the solution. It is a kind of efficiency coefficient, used to compare costs before and after introducing the process of optimization. We start with the situation as there is no optimization involved, and it is our initial state. If the ambulances each time start moving from the Central garage and visit all $\boldsymbol{n}$ destinations, we deal with total $\operatorname{cost} C_{\text {tot }}$ and it is sum of distances illustrated in Figure 1.

If we apply the achieved semi-optimal solution and cover all $\boldsymbol{n}$ destinations staring from the proposed optimal $\boldsymbol{p}$-parking places, we lowered total distance and total cost is now $C_{\text {opt }}$. We have now the case illustrated in Figure 2.

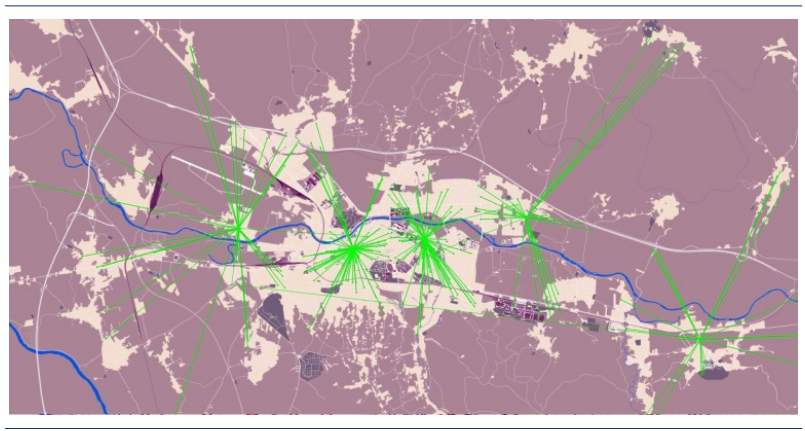

Figure 2. Total sum of distances according to the proposed solution $C_{o p t}$. 
The efficiency coefficient had to exclude the direct impact of the absolute values to its size. So, we proposed the following definition of "Saving ratio" as relative value, described in (16):

$$
S r=\frac{C_{o p t}}{C_{t o t}} \cdot 100(\%)
$$

The saving ratio $S_{r}$ gives us percentage in which we succeeded to decrease the cost of the initial solution. We assume that the initial solution represents $100 \%$ and $S_{r}$ gives gain in savings after we introduced semi-optimal solution.

\section{THE ANALYSIS OF OBTAINED RESULTS}

The comparative advantage of approach applied in this work is based on the fact that we deal with data obtained from the archived database connected to the AVL system, which has been in use for several years. Information about destinations in the particular case discussed in this work is extracted from the database where everyday drives of medical emergency vehicles were recorded during the period from September 01, 2014 until September 20, 2014. In that time frame, every step of the fleet of ambulances of the Emergency medical service in Niš was recorded. The analysis and algorithms which can be found in the literature available to these authors, related to similar optimization tasks, are commonly realized on the artificially generated input data, on the data of the population density, data from police reports, phone calls etc.

For the purpose of analysis of the impact of multiple coverage to the efficiency of generated solution, we analyzed the archived data and we chose time interval which delivered 218 destinations. We assumed that all vehicles travel from the Central garage of Emergency medical service and after intervention, the vehicles returns, using the same route. The starting $\operatorname{cost}\left(C_{t o t}\right)$ in the case of 218 destinations and the Euclidian distance between destinations is calculated, taking into account the geographical distribution of locations, and it is shown in Figure 3. The amount of total cost is $943.909,00$ meters.

After the second phase, we have populated the elements in the matrix with routed distances. We continue with same 218 destinations visited from the central garage, but now with the routed distances between destinations, and the total initial distance is now $990.431,00$ meters. As we expected, this value is larger than value of $C_{t o t}$, displayed in Figure 1 because moving by road network assumes longer way in comparision to the moving by hypothetic straight line. The third stage of algorithm is limited to 20 iterations and after calculations we get solution with the sum of total distances $441.007,00$ meters, which is represented by saving ratio approximately: $S_{r}=45 \%$.

During the fourth phase and the heuristic method based on Genetic algorithm, we introduce a single, double and triple coverage using the different mathematical formulation of the objective function represented in the expressions (1), (7) and (15). The construction of the basic elements of Genetic algorithm and structure of the fourth phase is available in [16], [17] and [18]. We had to mention only a couple of important hints: used structure of chromosomes is numerical, length of chromosome is defined with available total number of vehicles and objective function is routed distance needed to reach all destinations. Single, double and triple coverage required additional adaptation of the "fitness"-function, in accordance with the objectives and reached adaptation level of the offsprings.

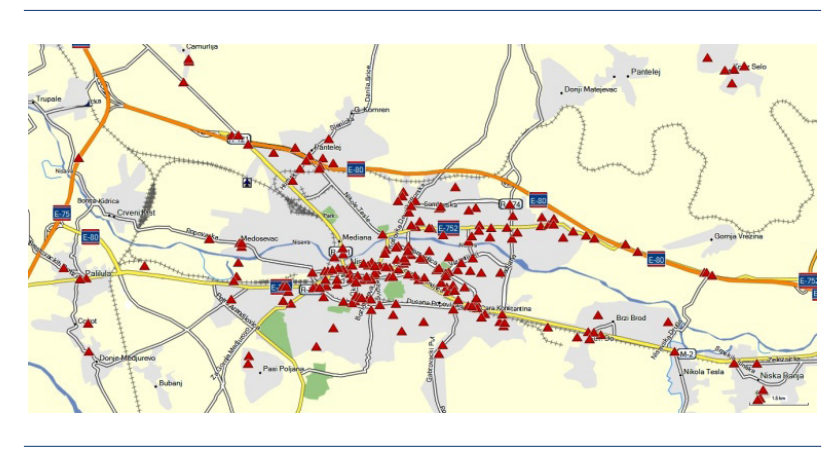

Figure 3. Layout of the 218-destinations $\left(\boldsymbol{C}_{t o t}\right)$.

At first glance, it is clear that in the case of double coverage, the number of simple mathematical operations required to calculate the amount of fitness-function practically doubles. Also, in the case of triple coverage, the number of operations is three times larger, as it is in the case of single coverage. The Genetic algorithm is limited and stops after 100 generations.

Figure 4 shows the layout of the parking places in the case of a single coverage of 218 destinations and 5 available vehicles. Figure 5 shows the solution obtained by the treatment of the same input data, but with a double coverage. Figure 6 presents the layout of the solution in the case of the triple coverage. Comparative observation of all three cases clearly shows that if we go from the single to the multiple coverage, the proposed parking places are concentrated close to the city core with smaller distance between parking places. This behavior of the 
obtained solutions is rather expected, because the largest concentration of destinations was in the city center, as can be seen in Figure 1.

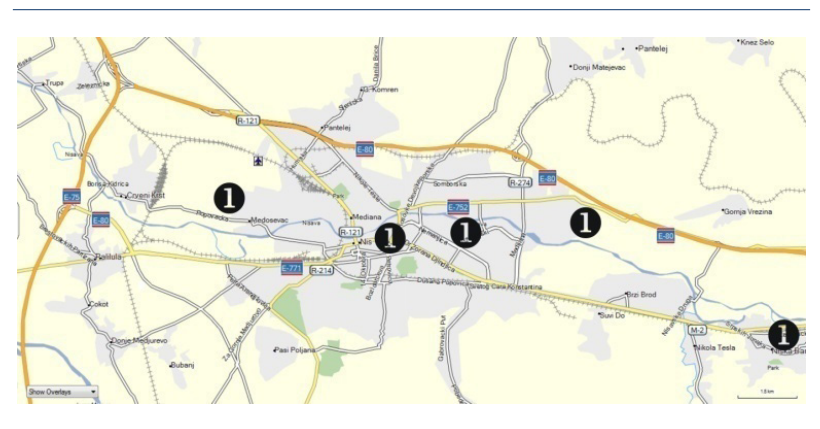

Figure 4. Layout of the $\boldsymbol{p}=5$ parking places - single coverage.

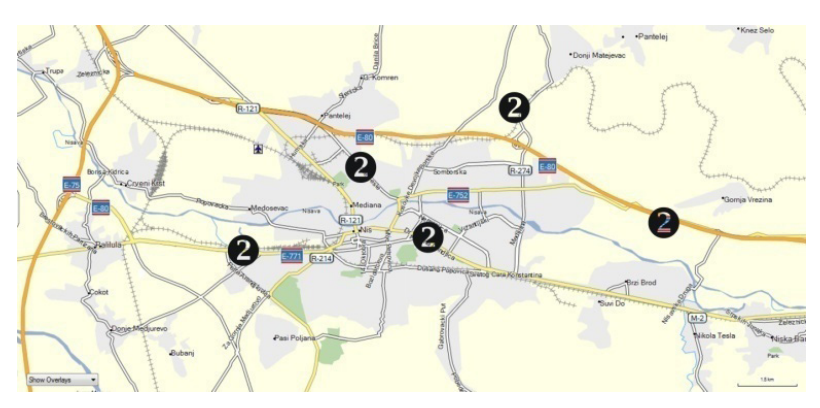

Figure 5. Layout of the $\boldsymbol{p}=5$ parking places - double coverage.

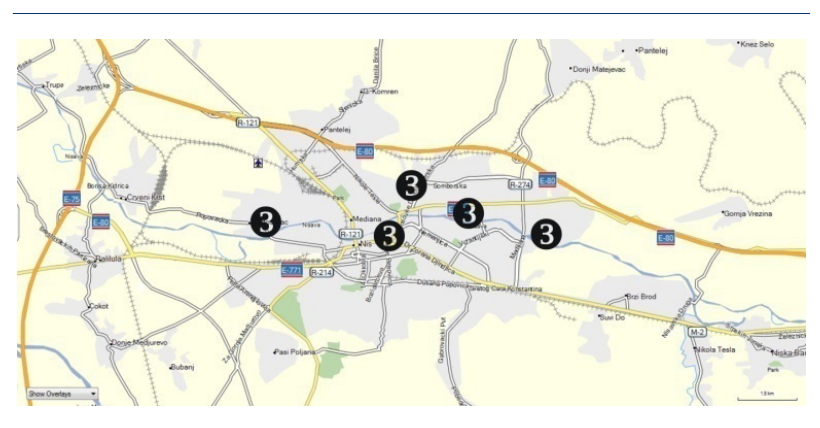

Figure 6. Layout of the $\boldsymbol{p}=5$ parking places - triple coverage.

Explanation of this trend lies in the simple fact that in the city core area, we find the greatest population density. With the introduction of four or five-fold coverage with $\boldsymbol{p}=5$ vehicles available, we approach to the concentration of all vehicles in the area of the central garage, and it was the initial set up, from which we started optimization process.

The data in Table I and graphic in Figure 7 demonstrate the behaviour of saving ratio (16) according to the execution of the algorithm in all three cases of different coverage. In Figure 7, we can see $S_{r}$ varies during the third stage of the algorithm and rough method of finding the initial solution, implemented during the first 20 iterations.

\begin{tabular}{lllllll}
\hline & \multicolumn{1}{c}{$\begin{array}{c}\text { Single } \\
\text { cover. }\end{array}$} & $\begin{array}{c}\text { Double } \\
\text { cover. }\end{array}$ & $\begin{array}{l}\text { Triple } \\
\text { cover. }\end{array}$ \\
\hline $\boldsymbol{d}(\mathrm{km})$ & $\boldsymbol{S}_{r}(\%)$ & $\boldsymbol{d}(\mathrm{km})$ & $\boldsymbol{S}_{r}(\%)$ & $\boldsymbol{d}(\mathrm{km})$ & $\boldsymbol{S}_{r}(\%)$ \\
\hline No opt. & 990.431 & $100 \%$ & 990.431 & $100 \%$ & 990.431 & $100 \%$ \\
\hline III phase & 441.007 & $45 \%$ & 614.067 & $62 \%$ & 693.302 & $70 \%$ \\
\hline IV phase 386.268 & $39 \%$ & 455.598 & $46 \%$ & 623.972 & $63 \%$ \\
\hline
\end{tabular}

Table I. Distances and saving ratio $\left(S_{r}\right)$

After the end of phase III, we enter into the phase in which the Genetic algorithm is executed in 100 generations. With each generation, solution is getting better and the algorithm quickly converges to the semi-optimal solution.

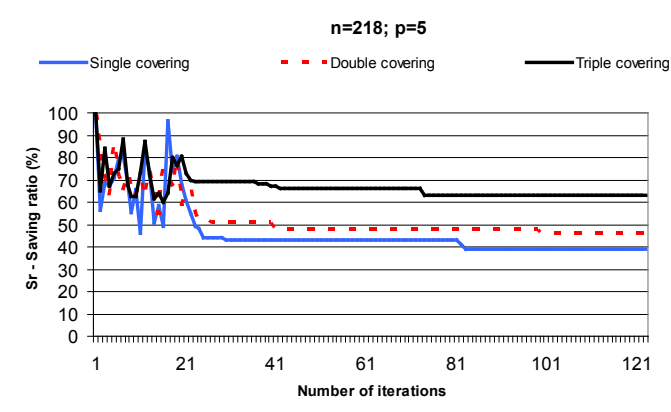

Figure 7. Behaviour of algorithm in cases of different coverage.

As we can see, $S_{r}$ is $39 \%$ for the case of a single coverage, and it is our solution with geographical layout shown in Figure 4. In the case of a double coverage and layout shown in Figure 5, the best $S_{r}$ is $46 \%$, while in the case of triple coverage and the layout shown in Figure 6, $S_{r}$ is $63 \%$. Table I shows the behavior of the total distance amount as the algorithm flows through certain stages.

\section{CONCLUSION}

If we monitor changes of the saving ratio when different coverage is used in the optimization process, some differences in the efficiency of solutions generated 
by differences in approach could be observed. However, if we compare single and double coverage, differences are not so significant in magnitude, and practical usefulness of this approach with double coverage has a lot of sense. This is particularly true if we take into account the statement that double coverage reflects the realistic needs of the observed service users.

Furthermore, multiple coverage does not have too much sense, especially if the size of the available fleet is small, as in our particular case, where we had only 5 vehicles available.

\section{REFERENCES}

[1] R.L. Church, C. ReVelle. The maximal covering location problem. Papers of the Regional Science Association 32, 101-118 (1974)

[2] K. Hogan, C. ReVelle. Concepts and applications of backup coverage. Management Science 34, 14341444 (1986)

[3] M. Gendreau, G. Laporte, F. Semet, 1997. Solving an ambulance location model by tabu search. Location Science 5 (2), 75-88.

[4] S.L. Hakimi, "Optimum Distribution of Switching Centers in a Communication Network and Some Related Graph Theoretic Problems", Operations Research, 13(3):462-475, 1965, [Online]. Available: http://dx.doi.org/10.1287/opre.13.3.462

[5] C.S. ReVelle, R.W. Swain, "Central Facilities Location", Geographical Analysis, Volume 2, Issue 1, pages 30-42, January 1970; [Online]. Available: DOI: 10.1111/j.1538-4632.1970.tb00142.x

[6] M.B. Teitz, P. Bart, "Heuristic Methods for Estimating the Generalized Vertex Median of a Weighted Graph", Operations Research, 16(5):955-961, 1968, [Online]. Available: http://dx.doi.org/10.1287/ opre.16.5.955

[7] P.J. Densham, G. Rushton, "Designing and implementing strategies for solving large location-allocation problems with heuristic methods", Technical Report 91-10, National Center for Geographic Information and Analysis, Buffalo, NY, 1991, [Online]. Available: doi: 10.1068/a240289

[8] E.L.F. Senne, L.A.N. Lorena, "Lagrangean/surrogate heuristics for p-median problems", Computing Tools for Modeling, Optimization and Simulation, 115-130, Springer US, 2000, [Online]. Available: http://citeseerx.ist.psu.edu/viewdoc/download?doi $=10.1 \cdot 1 \cdot 26.3011 \& \mathrm{rep}=\mathrm{rep} 1 \& \mathrm{type}=\mathrm{pdf}$

[9] P. Hansen, N. Mladenović, "Variable neighborhood search for the p-median", Location Science, 5(4):207-226, 1997, [Online]. Available: doi:10.1016/ S0966-8349(98)00030-8
[10] K.E. Rosing, C.S. ReVelle, "Heuristic concentration: Two stage solution construction", European Journal of Operational Research, Volume 97, Issue 1, 16 February 1997, Pages 75-86, [Online]. Available: doi:10.1016/S0377-2217(96)00100-2

[11] C. M. Hosage, M. F. Goodchild, "Discrete space location-allocation solutions from genetic algorithms", Annals of Operations Research, 6:35-46, 1986, [Online]. Available: http://www.geog.ucsb. edu/ good/ papers/78.pdf

[12] O. Alp, E. Erkut, Z. Drezner, "An efficient genetic algorithm for the p-median problem", Annals of Operations Research, vol. 122, pp. 21-42, 2003, [Online]. Available: http://link.springer.com/article /10.1023/ A:1026130003508

[13] P. Kolesar, W. Walker, J. Hausner, "Determining the Relation between Fire Engine Travel Times and Travel Distances in New York City", Operations Research, Vol. 23, No. 4, pp. 614-627, 1975, [Online]. Available: http://dx.doi.org/10.1287/opre.23.4.614

[14] S. Budge, A. Ingolfsson, D. Zerom, "Empirical Analysis of Ambulance Travel Times: The Case of Calgary Emergency Medical Services", Management Science, Vol. 56, No. 4, pp. 716-723, 2010, [Online]. Available: http://dx.doi.org/10.1287/ mnsc. 1090.1142

[15] M. Reinthaler, B. Nowotny, F. Weichenmeier, R. Hildebrandt, "Evaluation of speed estimation by floating car data within the research project DMotion", 14th World Congress on Intelligent Transport Systems, Beijing, China, 2007, [Online]. Available: http://www.dmotion.info/download/ ITS_Weichenmeier_full_paper.pdf

[16] Č. Vasić, D. Rančić, B. Predić, D. Mitić, “One Approach to Dynamical Relocation of Emergency Ambulance Vehicles Based on Geographic Information Systems", X Triennial International SAUM Conference on Systems, Automatic Control and Measurements, Niš, Serbia, November 10-12, 2010

[17] Č. Vasić, B. Predić, "Using GIS in Dynamic Relocation of Emergency Ambulance Vehicles", International Journal of Research and Reviews in Computer Science (IJRRCS), pp. 211 - 217, Vol. 2, No. 1, March 2011, [Online]. Available: http://www. scholarlyexchange.org/ojs/index.php/IJRRCS/article /wiev/7913

[18] Č. Vasić, B. Predić, D. Rančić, P. Spalević, Dž. Avdić, "Dynamic Relocation of Emergency Ambulance Vehicles Using the AVL Component of the GPS/GPRS Tracking System", Acta Polytechnica Hungarica, Volume 11, Number 9, 2014, [Online]. Available: DOI: 10.12700/APH.11.09.2014.09.3

[19] „Garmin BaseCamp, Ver.4.3.4“; Garmin Ltd; Lenexa, Kansas, USA 2008-2014; [Online]. Available: http://www.garmin.com 